\title{
Recent trends in treatment of hepatic non parasitic cystic lesions: Outcome of laparoscopic management
}

\author{
Ahmed H Ali, MD; Wafi Fouad Salib, MD; Ahmed Nafei, MD; \\ Mohamed A Nada, MD
}

\author{
Department of General Surgery, Ain Shams University, Cairo, Egypt.
}

\begin{abstract}
Background: Hepatic cystic lesions of non parasitic origin are mostly benign single, multiple, or diffuse cysts which contain clear serous fluid. Asymptomatic patients usually need no active treatment apart from follow up. In some patients, the cysts become painful and symptomatic which necessitate surgical intervention in the form of surgical deroofing or fenestration. Open surgical techniques used to be the treatment of choice since there is no harm to establish a free communication between the cyst and the peritoneal cavity because the cystic fluid contains no bile or microorganisms and its composition is similar to plasma. Recently, laparoscopic surgery is the treatment of choice as it can give similar results with less morbidity, and shorter hospital stay.

The aim of this study was to evaluate the postoperative morbidity and, the incidence of relapses in the laparoscopic treatment of non-parasitic hepatic cysts and with polycystic liver disease during one year of postoperative follow up.

Patients and methods: From March 2005 to September 2009, twenty patients (18 patients with simple non-parasitic hepatic cysts and 2 patients with polycystic liver disease (PCLD) with few large cysts in the anterior hepatic segments) of a mean age of 42 years (range 23-62) and of both sexes (16 women and 4 men) underwent laparoscopic fenestration and deroofing at Ain Shams University Hospitals. Before embarking on surgery, routine investigations, US, CT and serological tests for hydatid disease were requested. Tumour markers and bacteriological, chemical and cytological examination of the cystic fluid aspirated under US-guidance were requested if needed.

Results: Computed tomography with contrast enhancement revealed simple hepatic cysts in 18 cases with a mean diameter of $10 \mathrm{~cm}$ (range 5-14 cm). There were 8 in the left lobe (segment II and III) and 10 in the right hepatic lobe. Polycystic liver disease was seen in 2 cases with a mean cyst diameter of $8 \mathrm{~cm}$ (range 6-10 cm). The dominant cyst was in the right hepatic lobe in 1 case and in the left lobe in the other case. Serological test for hydatid was done and was negative for all cases. Laparoscopic deroofing was completed successfully in 16 cases and conversion was needed in 4 cases in which the cysts were deeply seated and inaccessible by the laparoscope. Open surgical deroofing was possible in 2 cases and left lateral segmentectomy was done in 2 cases. There was neither perisurgical mortality nor significant morbidity in this study. One year postoperative results showed recurrence of the cyst in two cases.

Conclusion: Laparoscopy can be considered a safe and efficacious treatment for non-parasitic hepatic cysts and with polycystic liver disease.
\end{abstract}

\section{Introduction:}

The liberal usage of non invasive imaging techniques has revealed that asymptomatic hepatic cysts are quite common occurring in up to $5-10 \%$ of the population, with a sharp rise in incidence with age. ${ }^{1}$ The pathogenesis is related to the type of cyst. Simple cysts may be solitary or multiple, are lined by cuboidal epithelium and are believed to arise from the abnormal development of intrahepatic bile ducts in utero. ${ }^{2}$ Polycystic liver disease is an autosomal dominant disease characterized by mutations on two genes on chromosome 19.3 Patients with polycystic liver disease may also 
have cysts in other organs, particularly the kidneys. Neoplastic cysts (benign biliary cystadenomas and biliary cystadenocarcinomas) are acquired, but the cause is unknown. Traumatic cysts are also acquired and result from bile leakage from an injured intrahepatic bile duct after trauma. Based on the aetiology of the liver cysts, many classifications are established, but the simple distinction between true cysts and pseudocysts is more sound. ${ }^{4}$ Pseudocysts lack a wall consisting of epithelial cells and originate from a traumatic lesion or from neoplastic growth. ${ }^{5}$ The majority of patients with non-parasitic hepatic cysts are asymptomatic and the cysts may be found incidentally at laparotomy or with abdominal imaging. Non parasitic liver cysts that are small and do not cause symptoms are considered benign and do not require treatment. However they should be observed for growth and checked for malignancy. $45 \%$ of patients are symptomatic and present with non-specific symptoms such as pain, nausea, vomiting, early satiety or heartburn. Rarely, cysts can present as a result of complications such as haemorrhage, rupture, infection, torsion, portal hypertension, malignancy and obstructive jaundice.$^{6-10}$ In symptomatic or complicated cases it is usual to intervene and the procedures available include percutaneous aspiration with ethanol injection, laparoscopic deroofing, open deroofing, hepatic resection and transplantation. There is considerable controversy as to which procedure is preferable. ${ }^{11}$ The technique of deroofing, sometimes referred to as fenestration, means drainage of the cyst content into the peritoneal cavity by excising part of the cyst wall. It is successfully applied to

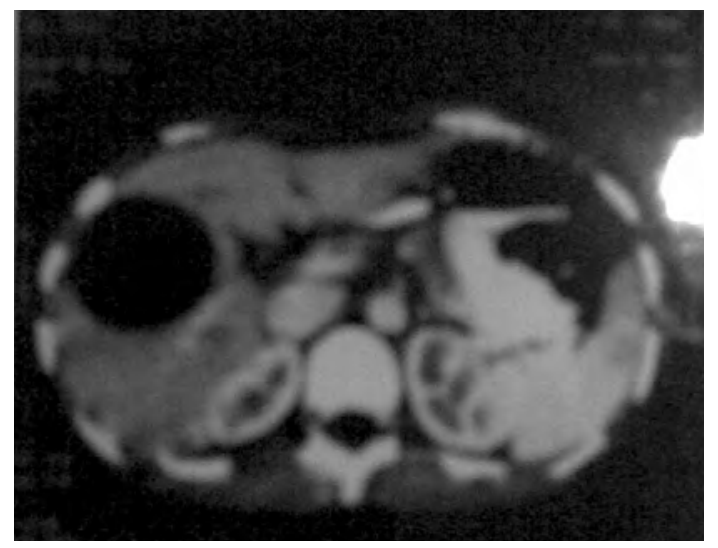

Figure (1): Simple solitary liver cyst. patients with simple cysts and those with polycystic liver disease. ${ }^{12}$ This procedure provides effective decompression and conserves almost all of the functional liver tissue. However, this intraperitoneal drainage is risky when the cyst contains infectious material or when there is biliary communication with the cyst. ${ }^{5}$ The application of the minimally invasive surgery in the recent era have revolutionized many fields of conventional surgery, and it was not surprising that hepatic cysts too have become the subject of laparoscopic surgery. ${ }^{13}$

Laparoscopic deroofing was first reported in 1991,14 and most studies seem to agree that it is the treatment of choice for simple cysts. However, recurrence rates vary greatly, from $0 \%$ to $25 \% .{ }^{14,15}$ Other studies have suggested that open deroofing and resection are better operations. ${ }^{16,17}$ The main goal of the laparoscopic approach is to achieve results comparable to the open surgical procedures with fewer complications and shorter hospital stay.

\section{Aim of the work:}

The aim of this work is to elicit the feasibility and the effectiveness of laparoscopic deroofing in the management of non parasitic liver cysts.

\section{Patients and methods:}

This study was conducted at Ain Shams University Hospitals between March 2005 to September 2009. Twenty patients with nonparasitic symptomatic simple solitary liver cysts Figure(1), and polycystic liver disease with a dominant cyst Figure(2) were included in the study.

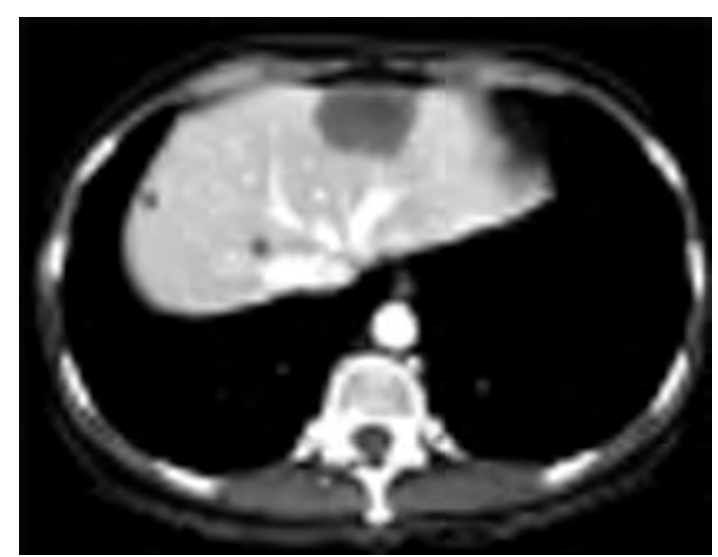

Figure (2): Polycystic liver with a dominant cyst. 
The following data were recorded for each patient:

1- Age and sex.

2- Presurgical symptoms.

3- Preoperative routine blood investigation results: Complete blood count (CBC), prothrombin time (PT), partial thromboplastin time (PTT), liver function tests including serum albumin, serum electrolytes, urea and creatinine.

4- Abdominal ultrasound.

5- Computed tomography with intravenous contrast enhancement to identify the site and number of cysts.

6- Serological tests for Hydatid.

7- Ultrasound guided aspiration sample of the cyst fluid if needed ensuring its clarity then submitting it to chemical, bacteriological and cytological examination.

8- Tumour markers if needed.

\section{Methods:}

The patients were scheduled essentially for laparoscopic deroofing of the simple hepatic cyst or the dominant cyst of polycystic liver disease, excising as much as possible of the accessible portion of the cyst. Conversion to open surgery using a right subcostal incision was needed when the cysts were difficult to access. A single dose of third generation cephalosporine was administered parentally with induction of anaesthesia for all patients. The laparoscopic access to the peritoneal cavity was achieved by inserting a $10 \mathrm{~mm}$ port at the umbilicus using the open technique. Insertion of further ports was governed by the precise location of the hepatic cyst. Decompression and aspiration of the content was performed to give an access to the superior aspect of the cyst and to facilitate mobilization and as radical excision as possible of its roof. The excised tissue was delivered through the nearest port to be submitted to histopathologic study. The remaining wall of the cyst was thoroughly inspected to exclude the presence of suspicious masses or nodules. Haemostasis was achieved with electro-cautery and clips. The operation was concluded by the insertion of a suction drain.

The patients were followed up as out- patient until relief of symptoms, first after one month then after 3 months them every 6 months with a mean follow- up of 12 months (9-15 months).

\section{Statistics:}

All data collected were analyzed using SPSS 11 for Windows statistical software.

\section{Results:}

In this study, 20 patients (16 women and 4 men) with a median age of 42 years (range 2362 ), underwent surgery for simple hepatic cysts and/or dominant cyst in polycystic disease of the liver. All patients were symptomatic, and pain was the commonest dominant presentation where it appeared in 18 patients of the 20 patients. Vomiting was the main presentation in 9 patients and abdominal swelling was present in 6 patients, Table(1). In all patients, the preoperative liver function tests were normal as well as other blood tests as the complete blood count and the total and differential leucocytic count. All patients underwent preoperative computerized tomography with intravenous contrast enhancement which revealed:

- Solitary simple cysts in 18 patients with a mean cyst diameter of $10 \mathrm{~cm}$ (range 5-14 $\mathrm{cm}$ ) and the cyst locations were: 8 in the left lobe of the liver (segment II and III) and 10 in the right hepatic lobe (segment V, VI,VII, and VIII).

- Polycystic liver disease was found in 2 cases with a mean cyst diameter of $8 \mathrm{~cm}$ (range $6-10 \mathrm{~cm}$ ). The dominant cyst was in the right lobe in one patient and in the left lobe in the other patient. 
Table (1): Patients presentation.

\begin{tabular}{|l|l|l|}
\hline Type of cyst & \multicolumn{1}{|c|}{ Simple } & \multicolumn{1}{c|}{ Polycystic } \\
\hline Number of patients & $\begin{array}{l}18(10 \text { in right lobe\&8 in } \\
\text { left lobe })\end{array}$ & $\begin{array}{l}\text { 2 (one in right lobe \& one in } \\
\text { left lobe) }\end{array}$ \\
\hline Mean age in years & $44(26-65)$ & $38(32-41)$ \\
\hline Sex ( Male/Female) & $4: 14$ & $0: 2$ \\
\hline Complaints & & \\
Pain & 2 & 1 \\
Pain + Swelling & 6 & 0 \\
Pain + Vomiting & 9 & 0 \\
Painless swelling & 1 & 1 \\
\hline Mean cyst diameter & $10 \mathrm{~cm}($ range $5-14 \mathrm{~cm})$ & $8 \mathrm{~cm}($ range $6-10 \mathrm{~cm})$ \\
\hline
\end{tabular}

All patients were prepared, consented and scheduled for initial laparoscopic exploration and deroofing. Laparoscopic deroofing of the hepatic cysts was successfully completed in 16 patients; 15 patients with simple solitary cysts and 1 patient with polycystic liver disease. The cysts were superficial and easily accessible. The laparoscopic operation was completed in a range of 35-60 minutes with a mean operative time of 47 minutes.
Conversion from the laparoscopic approach to open surgery was deemed necessary in 4 patients. Deroofing was completed through the open surgical technique in 2 patients with simple solitary cysts all located in the right hepatic lobe. In last two cases, the location of the cyst was deep inside the left lobe substance which necessitated open surgical resection of the left lobe, Table(2).

Table (2): Operative results.

\begin{tabular}{|l|c|c|c|}
\hline Number of patients & Laparoscopic deroofing & Open deroofing & Resection \\
\hline Simple cysts (18) & 15 & 2 & 1 \\
\hline Polycystic (2) & 1 & 0 & 1 \\
\hline Total (20) & 16 & 2 & 2 \\
\hline
\end{tabular}

Postoperative results \& follow up, Table(3):

- Laparoscopic deroofing (16 patients): Three patients developed umbilical port site superficial wound infection which responded well to daily dressing and antibiotics.

- Open surgical deroofing (2 patients): One patient developed superficial wound infection postoperatively which responded well to daily dressing and antibiotics.

- Open surgical resection (2 patients): One patient developed bile leak of $100 \mathrm{cc}$ daily for 7 days, then gradually decreased to an amount of less than $50 \mathrm{cc}$ daily and the suction drain was removed after 15 days.

There have been no immediate or late postoperative mortality on follow up of all patients and the histopathology results for the excised cyst wall assured the benign nature of the cystic lesions in all patients.

All patients were followed up in the surgery outpatient clinic for one year. We had two symptomatic recurrences after laparoscopic deroofing during the follow up period. One recurrence followed laparoscopic deroofing of a simple cyst. The recurrence manifested 3 months following the procedure. This case required open surgical deroofing for the management of the recurrence and the patient is currently free of symptoms.

The recurrence in the other case appeared after 8 months and the patient was suffering polycystic liver disease.The patient refused further surgery and was managed by CT-guided aspiration. 
Table (3): Post operative results \& complications.

\begin{tabular}{|l|cc|c|}
\hline \multirow{2}{*}{ Operation } & \multicolumn{2}{|c|}{ Complication } & Recurrence \\
& Wound infection & Bile leak & \\
\hline Laparoscopic Deroofing & 3 & 0 & 1 \\
- Simple Cyst & 0 & 0 & 1 \\
- Polycystic Liver & & & 0 \\
\hline Open Deroofing & 1 & 0 & 0 \\
- Simple Cyst & 0 & 0 & 0 \\
- Polycystic Liver & & & 0 \\
\hline Resection & 0 & 0 & 1 \\
- Simple Cyst & 0 & & 0 \\
- Polycystic Liver & & & \\
\hline
\end{tabular}

\section{Discussion:}

Because of the low incidence of symptomatic hepatic cysts in the general population, there has been no consensus on the optimal approach to management of cystic liver disease and therefore patients are managed on an individual basis. Several studies have attempted to address this problem, but numbers of patients and lengths of follow-up have varied significantly and results are sometimes conflicting. In the management algorithm for patients with cystic disease, the clinician must be certain of the diagnosis and determine the precise indication for surgery. It is widely accepted that symptoms are non-specific and the diagnosis is one of exclusion, which involves ruling out other significant pathology.

An additional concern is the presence of malignancy. Although the radiological characterization of cyst pathology before surgery is generally accurate, a high index of suspicion for the presence of a neoplastic cyst should be maintained. This differentiation is even more difficult for simple cysts presenting with intra-cyst haemorrhage and with unusual radiological appearances, which could be mistaken for cystadenomas. The second key question concerns the type of intervention, as several options are available. The choice may reflect the type and extent of cystic disease as well as the expertise and technology available locally. Percutaneous drainage and ethanol injection are increasingly used in some centers for patients with smaller simple cysts. The results are mixed and there appears to be a high recurrence rate. Aspiration used in combination with instillation of alcohol may be of value in patients with significant comorbidity or in those with a single small cyst which is symptomatic. ${ }^{18}$

In the current era of minimally invasive surgery, laparoscopic deroofing appears to be the preferred initial approach in most centers for the treatment of simple cysts. ${ }^{19-21}$ However, others prefer resection as the primary surgery and report no recurrence and a $17 \%$ morbidity rate. ${ }^{22}$ In this current study, we used the laparoscopic approach as an initial operation and we had a success rate of $80 \%$ while the conversion rate was $20 \%$. Meanwhile, we did not have any significant postoperative morbidity. The overall morbidity rate was $25 \%$ (3 patients $15 \%$ developed umbilical port site superficial wound infection, one patient $5 \%$ developed superficial wound infection which responded well to daily dressing and antibiotics and one patient $5 \%$ developed bile leak which responded to conservative management). In patients with polycystic liver disease (PCLD), the choice of initial surgical technique is even more controversial as a result of the size and distribution of the cysts as well as the severe distortion in liver anatomy. In an attempt to rationalize the treatment, Gigot et al., ${ }^{23}$ proposed a classification based on preoperative computed tomography (CT) scanning and suggested that therapy should be tailored to the type of disease. However, this classification 
is not widely used by radiologists and, therefore, the lack of uniform radiology reporting in the present series did not allow us to analyse the type of treatment and the Gigot staging. We should also point out that cyst size alone is of limited value when planning treatment strategy because multiple small cysts in a polycystic liver disease (PCLD) patient are as likely to require intervention as a large solitary cyst. Laparoscopic deroofing has been increasingly used in PCLD, despite a high recurrence rate. ${ }^{24,25}$ However, any decision favouring laparoscopic deroofing should be carefully considered and should weigh the extent of disease, patient co-morbidity and the high risk of recurrence against the likely extent of improvement in symptoms. In patients with a dominant cyst pattern, a laparoscopic or open deroofing should be the preferred initial treatment, taking into account all of the above factors, whereas resection should be reserved for those with a diffuse cyst pattern. ${ }^{25}$

\section{Conclusion:}

The majority of simple liver cysts can be managed by a laparoscopic approach but there is a definite role for open resection in selected patients. The management of nonparasitic cystic liver disease has evolved with the development of new surgical technologies, but remains challenging and offers a prospect of high recurrence rates. Treatment should be undertaken in specialized hepatobiliary units to ensure high rates of success.

\section{References:}

1- Caremani M, Vincenti A, Benci A, Sassoli S, Tacconi D: Echographic epidemiology of non-parasitic hepatic cysts. J Clin Ultrasound 1996; 21: 115-118.

2- Cowles RA, Mulholland MW: Solitary hepatic cysts. J Am Coll Surgeons 2000; 191(3): 311-321.

3- Drenth JPH, Martina JA, Van de Kerkhof R, Bonifacino JS, Jansen JB: Polycystic liver disease is a disorder of cotranslational protein processing. Trends Mol Med 2005; 11(1): 37-42.

4- Klingler PJ, Schmid T, Bonder E, Schwelberger: Treatment of hepatic cysts in the era of laparoscopic surgery. $\mathrm{Br} J$ Surg 1997; 84: 438-444.
5- Litwin DEM, Taylor BR, Langer B, Greig $\mathrm{P}$ : Nonparasitic cysts of the liver. The case for conservative surgical management. Ann Surg 1987; 205: 45-48.

6- Chan CY, Tan $\mathrm{CH}$, Chew SP, Teh $\mathrm{CH}$ : Laparoscopic fenestration of a simple hepatic cysts. Singapore Med J 42(6): 268270.

7- Gesundheit N, Kent DL, Fawcett HD, Effron MK, Maffly RH: Infected liver cyst in a patient with polycystic kidney disease. West J Med 1982; 136: 246-249.

8- Davis CK, Schoffstall RO, Glass TF: Fatal complication of hepatic cystic disease. South Med J 1981; 74: 1409-1411.

9- Ratcliffe PJ, Reeders S, Theaker JM: Bleeding oesophageal varices and hepatic dysfunction in adult polycystic kidney disease. Br Med J 1984; 288: 1330-1331.

10-Cappell MS: Obstructive jaundice from benign, nonparasitic hepatic cysts: Identification of risk factors and percutaneous aspiration for diagnosis and treatment. Am J Gastroenterol 1988; 83: 93-96.

11-Sanchez H, Gagner M, Rossi RL, Jenkins RL, Lewis WD, Munson JL, et al: Surgical management of nonparasitic cystic liver disease. Am J Surg 1991; 161: 113-119.

12-Vauthey JN, Madden Gj, Blumgart LH: Adult polycystic disease of the liver. $\mathrm{Br} \mathrm{J}$ Surg 1991; 78: 524-527.

13-Gigot JF, legrand M, Hubbens G, Lee KT, Sheen PC : Laparoscopic treatment of non parasitic liver disease cysts: Adequate selection of patients and surgical technique. World J Surg 1996; 20: 556-561.

14-Paterson-Brown S, Garden OJ: Laserassisted laparoscopic excision of liver cyst. Br J Surg 1991; 78(9): 1047.

15-Zalaba Z, Tihanyi TF, Winternitz T, Nehez L, Flautner L: The laparoscopic treatment of non-parasitic liver cysts. Five years experience. Acta Chir Hung 1999; 38(2): 221-223.

16-Pitale A, Bohra AK, Diamond T: Management of symptomatic liver cysts. Ulster Med J 2002; 71(2): 106-110.

17-Tocchi A, Mazzoni G, Costa G, Cassini D, Bettelli E, Agostini N, et al: Symptomatic nonparasitic hepatic cysts. Arch Surg 1(37): 154-158. 
18- Gall TMH, Oniscu GC, Madhavan K, Parks RW, Garden OJ: HPB 2009; 11(3): $235-$ 241.

19-Regev A, Reddy KR, Berho M, Sleeman D, Levi JU, Livingstone AS, et al: Large cystic lesions of the liver in adults: A 15year experience in a tertiary center. Am Coll Surg 2001; 193: 36-45.

20-Koperna T, Vogl S, Satzinger U, Schulz F: Nonparasitic cysts of the liver: Results and options of surgical treatment. World J Surg 1997; 21: 850-855.

21-Gigot JF, Metairie S, Etienne J, Horsmans Y, van Beers BE, Sempoux C, et al: The surgical management of congenital liver cysts. Surg Endosc 2001; 15(4): 357-363.

22-Tocchi A, Mazzoni G, Costa G, Cassini D, Bettelli E, Agostini N, et al: Symptomatic nonparasitic hepatic cysts. Arch Surg 2002; 137: 154-158.
23-Gigot JF, Jadoul P, Que F, van Beers BE, Etienne J, Horsmans Y, et al: Adult polycystic liver disease: Is fenestration the most adequate operation for long-term management? Ann Surg 1997; 225: 286294.

24-Hansman MF, Ryan JA Jr, Holmes JH 4th, Hogan S, Lee FT, Kramer D, et al: Management and long-term follow-up of hepatic cysts. Am J Surg 2001; 181: 404410.

25-Tan YM, Ooi LL: Highly symptomatic adult polycystic liver disease: Options and results of surgical management. ANZ $J$ Surg 2004; 74: 653- 657. 